\title{
ON THE REGULARITY OF CROSSED PRODUCTS
}

\author{
V. BOVDI, S. MIHOVSKI
}

\begin{abstract}
We study some generalizations of the notion of regular crossed products $K * G$. For the case when $K$ is an algebraically closed field, we give necessary and sufficient conditions for the twisted group ring $K * G$ to be an $n$-weakly regular ring, a $\xi^{*} N$-ring or a ring without nilpotent elements.
\end{abstract}

\section{INTRODUCTION}

Let $G$ be a group, $U(K)$ the group of units of the associative ring $K$ with identity and let $\sigma: G \rightarrow \operatorname{Aut}(K)$ be a map of $G$ into the group $\operatorname{Aut}(K)$ of automorphisms of $K$. Let $K * G=K_{\rho}^{\sigma} G=\left\{\sum_{g \in G} u_{g} \alpha_{g} \mid \alpha_{g} \in K\right\}$ be the crossed product (in the sense of [1), of the group $G$ over the ring $K$ with respect to the factor system

$$
\rho=\{\rho(g, h) \in U(K) \mid g, h \in G\}
$$

and the map $\sigma: G \rightarrow \operatorname{Aut}(K)$. Moreover we assume that the factor system $\rho$ is normalized, i.e. $\rho(g, 1)=\rho(1, g)=\rho(1,1)=1$ for any $g \in G$.

In particular, if $\sigma=1$, then the crossed product $K * G$ is called a twisted group ring, which we denote by $K_{\rho} G$. If the factor system $\rho$ is unitary, i.e. $\rho(g, h)=1$ for all $g, h \in G$, then $K * G$ is called a skew group ring and is denoted by $K^{\sigma} G$. In the case, when $\rho=1$ and $\sigma=1$, then $K * G$ is the ordinary group $\operatorname{ring} K G$.

In the present paper we study properties of crossed products $K * G$ which are generalizations of the notion of a regular ring. For the case when $K * G$ is a twisted group ring over the algebraically closed field $K$, we give necessary and sufficient conditions for $K * G$ to be an $n$-weakly regular ring $(n \geq 2)$, a $\xi^{*} N$-ring or a ring without nilpotent elements. Our investigation can be considered as a generalization of certain results of [2, 3, 4, 7, 11, 12, earlier obtained for group rings. Note that we exclude the case when $K * G$ is a skew group ring, so we do not cite any reference from that topic.

\section{TWisted GRoup ALGEBRAS Without NiLPotent ELEMENTS}

Denote the $K$-basis of $K * G$ by $U_{G}=\left\{u_{g} \mid g \in G\right\}$. The multiplication of $u_{g}, u_{h} \in U_{G}$ is defined by $u_{g} u_{h}=\rho(g, h) u_{g h}$, where $\rho(g, h) \in \rho$ and $g, h \in G$. The factor system $\rho$ of the crossed product $K * G$ is called symmetric, if for all elements $g, h \in G$ the condition $g h=h g$ yields $\rho(g, h)=\rho(h, g)$. The finite subset $\operatorname{Supp}(a)=\left\{g \in G \mid \alpha_{g} \neq 0\right\}$ of $G$ is called the support of the element $a \in K * G$.

We shall freely use the following.

Lemma 1. Let $K * G$ be a crossed product and suppose that axb $=c$ for some $x, a, b, c \in K * G$. If $H$ is the subgroup of $G$ generated by $\operatorname{Supp}(a), \operatorname{Supp}(b)$ and $\operatorname{Supp}(c)$, then there exists an element $y \in K * H$, such that ayb $=c$.

2010 Mathematics Subject Classification. Primary: 16S35; Secondary: 20C07, 16S34, 16E50.

Key words and phrases. crossed product, twisted group ring, regular ring. 
Proof. Indeed, if $x=y+z$, then $a y b+a z b=c$, where $y=\sum_{h \in H} u_{h} \alpha_{h}$ and $z=\sum_{g \notin H} u_{g} \beta_{g}$. This shows that $\operatorname{Supp}(a z b) \subseteq H$. Since $f g h \notin H$ for $f \in \operatorname{Supp}(a)$, $g \in \operatorname{Supp}(z)$ and $h \in \operatorname{Supp}(b)$, we conclude that $a z b=0$ and $a y b=c$, as it was requested.

Corollary 1. If $g \in G$ has infinite order, then $u_{g}-1$ is neither a one-sided zero divisor, nor a one-sided invertible element of the crossed product $K * G$.

Proof. In fact, if $u_{g}-1$ is either a one-sided zero divisor, or a one-sided invertible element of $K * G$, then by Lemma 1 we may assume that $u_{g}-u_{1}$ is also such an element of $K * H$, where $H=\langle g\rangle$ is an infinite cyclic group. But $H$ is an ordered group, a contradiction.

For twisted group algebras we give a refinement of Corollary 2 and Lemma 2 of [7] (see p.68) which were earlier proved for group rings.

Theorem 1. Let $K_{\rho} G$ be a twisted group algebra of a torsion group $G$ over the algebraically closed field $K$. The ring $K_{\rho} G$ does not contain nilpotent elements if and only if the following conditions hold:

(i) $G$ is an abelian group;

(ii) the order of every elements in $G$ is invertible in $K$;

(iii) the factor system $\rho$ is symmetric.

Proof. Assume that the conditions (i), (ii) and (iii) hold. Then the twisted group ring $K_{\rho} G$ is commutative. If $x \in K_{\rho} G$ is a nonzero nilpotent element and $H=$ $\langle\operatorname{Supp}(x)\rangle$, we conclude that $K_{\rho} H$ is a commutative artinian ring with a nonzero nilpotent element $x$. So, by Theorem 2.2 of $(8$, p.415), we get a contradiction.

Conversely, let $K_{\rho} G$ be a twisted group ring without nilpotent elements. If $g \in G$ is of order $n$ and $u_{g}^{n}=u_{1} \alpha_{g}$, where $\alpha_{g} \in U(K)$, then there exists an element $\mu_{g} \in U(K)$ such that $\mu_{g}^{n}=\alpha_{g}^{-1}$, because $K$ is algebraically closed. So for the element $v_{g}=u_{g} \mu_{g}$ we have $v_{g}^{n}=1$. Obviously,

$$
x=\left(v_{g}-1\right) u_{h}\left(1+v_{g}+v_{g}^{2}+\cdots+v_{g}^{n-1}\right)
$$

is a nilpotent element of $K_{\rho} G$ for all $h \in G$ as far as $x^{2}=0$. Thus $x=0$, so we conclude that

$$
u_{h}=v_{g} u_{h} v_{g}^{i} \quad(0 \leq i \leq n-1) .
$$

Examining the supports we can deduce that $h^{-1} g h=g^{-i} \quad(h \in G)$. Therefore all cyclic subgroups of $G$ are normal. This implies that $G$ is either abelian or hamiltonian. If $g h=h g$, then $i=n-1$ and by (11) it follows that $u_{h} v_{g}=v_{g} u_{h}$, since $v_{g}^{n}=1$ is the identity element of $K_{\rho} G$. So we conclude that $\rho(g, h)=\rho(h, g)$, i.e. the factor system $\rho$ is symmetric and condition (iii) holds.

If $\operatorname{char}(K)=p>0$ and $G$ contains an element $g$ of order $p$, then

$$
\left(1+v_{g}+v_{g}^{2}+\cdots+v_{g}^{p-1}\right)^{p}=0
$$

and we get a contradiction. This implies that condition (ii) also follows.

Assume that $G$ is hamiltonian and $\left\langle g, h \mid g^{4}=h^{4}=1, g^{2}=h^{2}, g^{h}=g^{-1}\right\rangle \cong Q_{8}$ is the quaternion group of order 8 . Then $h^{-1} g h=g^{-1}$ and $i=1$. Therefore in this case by (1) we have $u_{h}=v_{g} u_{h} v_{g}$, i.e.

$$
v_{h}=v_{g} v_{h} v_{g}
$$


where $v_{h}=u_{h} \mu_{h}$ and $v_{g}^{4}=v_{h}^{4}=1$. Since $G$ contains 2-elements, it follows from (ii) that $\operatorname{char}(K) \neq 2$.

$K$ being an algebraically closed field, it is clear that there exist nonzero elements $\alpha, \beta \in K$ for which $\alpha^{2}+\beta^{2}=0$. Then by (2) it is easy to verify that

$$
w=\alpha\left(v_{g}^{2} v_{h}-v_{h}\right)+\beta\left(v_{g}^{3} v_{h}-v_{g} v_{h}\right)
$$

is a nonzero nilpotent element of $K_{\rho} G$.

Indeed, $h \in \operatorname{Supp}\left(\alpha\left(v_{g}^{2} v_{h}-v_{h}\right)\right)$, but $h \notin \operatorname{Supp}\left(\beta\left(v_{g}^{3} v_{h}-v_{g} v_{h}\right)\right)$. Thus we have $w \neq 0$. Moreover, by (2) we obtain that $u_{h}^{2} v_{g}=v_{g} u_{h}^{2}$ and $u_{h} v_{g}^{2}=v_{g}^{2} u_{h}$. Then $w^{2}=\left(v_{g}^{2}-1\right)^{2}\left(\alpha v_{h}+\beta v_{g} v_{h}\right)^{2}$. Since $\left(v_{g}^{2}-1\right)^{2}=2\left(1-v_{g}^{2}\right)$ and

$$
\begin{aligned}
\left(\alpha v_{h}+\beta v_{g} v_{h}\right)^{2}=\left(\alpha^{2}+\beta^{2}\right) v_{h}^{2} & +\alpha \beta v_{h}^{2}\left(v_{g}^{2}+1\right) v_{g} \\
& =\alpha \beta v_{h}^{2}\left(v_{g}^{2}+1\right) v_{g},
\end{aligned}
$$

we obtain $w^{2}=2\left(1-v_{g}^{2}\right) \alpha \beta v_{h}^{2}\left(1+v_{g}^{2}\right) v_{g}=0$, which is impossible. Hence condition (i) follows, as requested.

\section{Regular CROSSED PROduCts}

An associative ring $R$ with unity is called regular (strongly regular) if for every $a \in R$ there is an element $b \in R$, such that $a b a=a$ ( $b a^{2}=a$, respectively). A ring $R$ is called $\xi^{*}$-ring ( $\xi^{*} N$-ring) if for every $a \in R$ there exists $b \in R$ such that $a b a-a$ is a central (central nilpotent, respectively) element of $R$. It is clear that every regular ring is a $\xi^{*} N$-ring and every $\xi^{*} N$-ring is a $\xi^{*}$-ring (see [7, 12]).

By the theorem of Auslander, Connell and Willamayor (see [3], Theorem 3, p.660), it is well known that a group ring is regular if and only if $K$ is regular, $G$ is a locally finite group and the order of every element $g \in G$ is invertible in $K$.

Our first result for this section is the following.

Theorem 2. Let $K * G$ be a crossed product of the group $G$ over the ring $K$ such that one of the following conditions is satisfied:

(i) $K * G$ is a $\xi^{*} N$-ring;

(ii) $K * G$ is n-weakly regular.

Then $G$ is a torsion group.

Proof. (i) Suppose that $g \in G$ is an element of infinite order. Then there exists a $b \in K * G$ and a natural number $n \geq 1$ such that

$$
x=\left(u_{g}-1\right) b\left(u_{g}-1\right)-\left(u_{g}-1\right)
$$

is a central element of $K * G$ and $x^{n}=0$. If $n=1$, then $x=0$ and

$$
\left(u_{g}-1\right)\left[b\left(u_{g}-1\right)-1\right]=0 .
$$

Since, by Corollary 1 the element $u_{g}-1$ is not a left zero divisor in $K * G$, we obtain that $b\left(u_{g}-1\right)=1$, i.e. $u_{g}-1$ is a left invertible element in $K * G$, which is also impossible. Therefore $n>1$ and

$$
x^{n}=\left(u_{g}-1\right)\left[b\left(u_{g}-1\right)-1\right] x^{n-1}=0 .
$$

In the same way we obtain that $z_{1}=\left[b\left(u_{g}-1\right)-1\right] x^{n-1}=0$. Suppose that for some $k \geq 1$ we have $z_{k}=\left[b\left(u_{g}-1\right)-1\right]^{k} x^{n-k}=0$. If $1<k<n$, as far as $x$ is 
central,

$$
\begin{aligned}
z_{k} & =x\left[b\left(u_{g}-1\right)-1\right]^{k} x^{n-k-1} \\
& =\left(u_{g}-1\right)\left[b\left(u_{g}-1\right)-1\right]^{k+1} x^{n-k-1}=0 .
\end{aligned}
$$

Now applying Corollary 1 we obtain that

$$
z_{k+1}=\left[b\left(u_{g}-1\right)-1\right]^{k+1} x^{n-k-1}=0 .
$$

Thus, by induction we conclude that $z_{n}=\left[b\left(u_{g}-1\right)-1\right]^{n}=0$.

The last equality shows that there exists $z \in K * G$ such that $z\left(u_{g}-1\right)=1$, which, by Corollary 1, is impossible.

(ii) Suppose that $g \in G$ is an element of infinite order. Then for some $b, c \in K * G$ we have $u_{g}-1=\left(u_{g}-1\right) b\left(u_{g}-1\right)^{n} c$. By Corollary 1 we have

$$
\left(u_{g}-1\right)\left[1-b\left(u_{g}-1\right)^{n} c\right]=0,
$$

we conclude that $b\left(u_{g}-1\right)^{n} c=1$. Hence it follows that $b\left(u_{g}-1\right) x=1$, where $x=\left(u_{g}-1\right)^{n-1} c$. If $e=x b\left(u_{g}-1\right)$, then

$$
e^{2}=x\left[b\left(u_{g}-1\right) x\right] b\left(u_{g}-1\right)=x b\left(u_{g}-1\right)=e,
$$

i.e. $e$ is a central idempotent of $K * G$. Thus we have

$$
\begin{aligned}
1=b\left(u_{g}-1\right) x & =b\left(u_{g}-1\right)\left[x b\left(u_{g}-1\right)\right] x \\
& =x b\left(u_{g}-1\right)\left[b\left(u_{g}-1\right) x\right]=x b\left(u_{g}-1\right),
\end{aligned}
$$

i.e. $u_{g}-1$ has a left invertible element $x b \in K * G$. Now again by Corollary 1 we obtain a contradiction, so the proof is complete.

Corollary 2. If the crossed product $K * G$ is a regular ring, then $K$ is also a regular ring and $G$ is a torsion group.

Proof. The claim follows from Theorem 2 and Lemma 1 .

Observe that the theorem of Auslander, Connell and Willamayor (see [3], Theorem 3, p.660) does not apply for crossed products. Indeed, if $K$ is a non-perfect field of characteristic $p>0$ and $G$ is the $p^{\infty}$-group, then there exists a twisted group ring $K_{\rho} G$, which must be a field (see [9], Proposition 4.2).

If $G$ satisfies the maximum condition for finite normal subgroups and the group ring $K G$ is a $\xi^{*} N$-ring, then $G$ is locally finite (see [11], Theorem 3, p.16).

We shall prove the locally finiteness of $G$ without the assumption of the maximum condition when $K$ is a field. First we recall that (see [10], p.308)

$$
\Delta(G)=\left\{g \in G \mid\left[G: C_{G}(g)\right]<\infty\right\}
$$

is a subgroup of $G$, where $C_{G}(g)$ is the centralizer of $g$ in $G$. Furthermore, we put

$$
\left.\Delta^{p}(G)=\langle g \in \Delta(G)| g \quad \text { is a } p \text {-element }\right\rangle,
$$

that is the subgroup of $\Delta(G)$ which is generated by all $p$-elements of $\Delta(G)$.

Now we are ready to prove the following.

Theorem 3. Let $K G$ be the group algebra of a group $G$ over a field $K$. If $K G$ is a $\xi^{*} N$-ring, then $G$ is a locally finite group. Moreover, if $\operatorname{char}(K)=p>0$ then $\Delta^{p}(G)$ contains all p-elements of $G$. 
Proof. Let $\mathfrak{N}(K G)$ be the union of all nilpotent ideals of $K G$. In particular, the central nilpotent elements of $K G$ are in $\mathfrak{N}(K G)$ and, consequently, $K G / \mathfrak{N}(K G)$ is a regular ring.

Assume $\operatorname{char}(K)=p>0$. By Theorem $8.19([10$, p.309 $)$,

$$
\mathfrak{N}(K G)=\mathfrak{R a d}\left(K\left[\Delta^{p}(G)\right]\right) K G,
$$

where $\mathfrak{R a d}\left(K\left[\Delta^{p}(G)\right]\right)$ is the Jacobson radical of the group ring $K\left[\Delta^{p}(G)\right]$. Obviously, the augmentation ideal $\omega\left(K\left[\Delta^{p}(G)\right]\right)$ is a maximal ideal of $K\left[\Delta^{p}(G)\right]$, so

$$
\mathfrak{N}(K G)=\mathfrak{R a d}\left(K\left[\Delta^{p}(G)\right]\right) K G \subseteq \omega\left(K\left[\Delta^{p}(G)\right]\right) K G .
$$

It is well-known (see [3], Theorem 3, p.660) that

$$
K\left[G /\left(\Delta^{p}(G)\right)\right] \cong K G / \omega\left(K\left[\Delta^{p}(G)\right]\right) K G
$$

and therefore the group algebra $K\left[G / \Delta^{p}(G)\right]$ is regular, as a homomorphic image of $K G / \mathfrak{N}(K G)$. This implies, by the theorem of Auslander, Connell and Willamayor (see [3], Theorem 3, p.660), that $G / \Delta^{p}(G)$ is locally finite and has no $p$-element. Thus we obtain that $\Delta^{p}(G)$ contains all the $p$-elements of $G$ and the group $G$ is locally finite (see [5], Theorem 23.1.1, p.215).

If $\operatorname{char}(K)=0$, then $\mathfrak{N}(K G)=0$ and $K G$ is regular. According to AuslanderConnell-Villamayor's theorem the proof is complete.

\section{4. n-WEAKLY REGULAR TWISTED GROUP ALGEBRAS}

Let $n \geq 2$ be a fixed natural number. A ring $R$ is called $n$-weakly regular [4] if for every $a \in R$ there exist elements $b, c \in R$ such that $a=a b a^{n} c$.

Obviously, an $n$-weakly regular ring $R$ has no nonzero nilpotent element. Indeed, if $R$ contains a nonzero nilpotent element, then there exists a nonzero nilpotent element $a \in R$ with $a^{2}=0$. Hence $a=a b a^{n} c=0$, which is impossible. From this fact we can conclude that all idempotents of an $n$-weakly regular ring are central.

In 2] (Theorem 2, p.119) it was proved that the group algebra $K G$ over a field $K$ is $n$-weakly regular $(n \geq 2)$ if and only if $K$ and $G$ satisfy at least one of the following two conditions:

(i) $\operatorname{char}(K)=p>0$ and $G$ is an abelian torsion group without $p$-elements;

(ii) $\operatorname{char}(K)=0$ and $G$ is either an abelian torsion group or a hamiltonian $\operatorname{gr} G=Q \times E \times A$, where $A$ is an abelian torsion group without 2-elements and the equation $x^{2}+y^{2}+z^{2}=0$ in $K A$ has only the trivial solution.

In the case when $K$ is an algebraically close field, this result can be extended to.

Theorem 4. A twisted group algebra $K_{\rho} G$ of a group $G$ over the algebraically closed field $K$ is $n$-weakly regular $(n \geq 2)$ if and only if the following conditions hold:

(i) $G$ is an abelian torsion group;

(ii) the order of every element of $G$ is invertible in $K$;

(iii) the factor system $\rho$ is symmetric.

Proof. Suppose that $K_{\rho} G$ is $n$-weakly regular. Then conditions (i), (ii) and (iii) hold by Theorems 1 and 2 ,

Conversely, if $K$ and $G$ satisfy the conditions (i), (ii) and (iii), then $K_{\rho} G$ is a commutative ring. Let $a \in K_{\rho} G$ be an arbitrary element. Then $a \in K_{\rho} H$, where $H=\langle\operatorname{Supp}(a)\rangle$ is a finite abelian group. Since $K_{\rho} H$ is a commutative semisimple artinian ring ([8], Theorem 2.2), we conclude that $K_{\rho} H$ is a direct product of 
fields, so $K_{\rho} H$ is $n$-weakly regular. This implies that $K_{\rho} G$ is $n$-weakly regular, as requested.

Analyzing the result of [2] (see Theorem 2, p.119) on $n$-weakly regular group rings and [7] (see Corollary 2, p.70) about strongly regular group rings we deduce that when $K$ is a field, then these two classes coincide.

In the case of twisted group algebras over an algebraically closed basic field we have the following.

Corollary 3. Let $K_{\rho} G$ be a twisted group algebra of a group $G$ over an algebraically closed field $K$. The following statements are equivalent:

(i) $K_{\rho} G$ is strongly regular;

(ii) $K_{\rho} G$ is $n$-weakly regular for every natural number $n \geq 2$;

(iii) $K_{\rho} G$ is $n$-weakly regular for some natural number $n \geq 2$;

(iv) $G$ is an abelian torsion group, the order of every element of $G$ is invertible in $K$ and the factor system $\rho$ is symmetric.

Proof. Suppose that $K_{\rho} G$ is a strongly regular ring. If $a \in K_{\rho} G$ and $a=a^{2} b$, then $a=a b a$, because $K_{\rho} G$ does not contain nilpotent elements. Now by induction it follows that $a=a b^{n} c$ for some $c \in K_{\rho} G$ and for every natural number $n \geq 1$. So (i) implies (ii) and, obviously, (ii) implies (iii). By the preceding theorem, (iii) implies (iv). Finally, by the Auslander-Connell-Villamayor theorem and by (iv) it follows that $K_{\rho} G$ is a commutative von Neumann ring and so (iv) implies (i).

\section{5. $\xi N$-TWISTED GROUP ALGEBRAS}

A ring $R$ is called a $\xi N$-ring if for any $a \in R$ there exists $b \in R$ such that $a^{2} b-a$ is a central nilpotent element of $R$ (see [11]).

Obviously, every $\xi N$-ring is a $\xi$-ring and, therefore, (see [6], Theorem 1, p.714) we deduce that every $\xi N$-ring is a $\xi^{*} N$-ring. Moreover, (see [6], Lemma 2, p.715) it follows that in $\xi N$-rings all nilpotent elements are central.

$\xi N$-group rings over commutative rings are described in [11] (Theorem 2, p.15). From this description, it follows that a group ring $K G$ over a field $K$ of characteristic $p>0$ is a $\xi N$-ring if and only if $G$ is an abelian torsion group.

Finally we prove the following.

Theorem 5. A twisted group algebra $K_{\rho} G$ of a group $G$ over the algebraically closed field $K$ is a $\xi N$-ring if and only if the following conditions hold:

(i) $G$ is an abelian torsion group;

(ii) the factor system $\rho$ is symmetric.

Proof. Let $K_{\rho} G$ be a $\xi N$-ring. Then ([6], Theorem 1, p.714) the $\operatorname{ring} K_{\rho} G$ is a $\xi^{*} N$-ring and, in view of Theorem 2, we conclude that $G$ is a torsion group. As far as $K$ is an algebraically closed field, for every element $g \in G$ of order $n$ there exists an $\mu_{g} \in U(K)$, such that $v_{g}=u_{g} \mu_{g} \quad\left(u_{g} \in U_{G}\right)$ and $v_{g}^{n}=1$. Then we put

$$
z=\left(v_{g}-1\right) v_{h}\left(1+v_{g}+v_{g}^{2}+\cdots+v_{g}^{n-1}\right), \quad(h \in H) .
$$


Clearly, $z^{2}=0$ and therefore $z$ is a central element of $K_{\rho} G$. Thus $z v_{h}=v_{h} z$ and, so we obtain the equality

$$
\begin{aligned}
2 v_{h} v_{g} v_{h}+\sum_{i=1}^{n-1} v_{g}^{i} v_{h} v_{g} v_{h} & +\sum_{i=2}^{n-1} v_{h} v_{g}^{i} v_{h} \\
& =\sum_{i=1}^{n-1} v_{g}^{i} v_{h}^{2}+\sum_{i=0}^{n-1} v_{h} v_{g}^{i} v_{h} v_{g} .
\end{aligned}
$$

If $\operatorname{char}(K)=2$, then $2 v_{h} v_{g} v_{h}=0$. Consequently for the product $v_{h} v_{g}^{2} v_{h}$ and for the corresponding supports we obtain the following three cases:

(A1) $v_{h} v_{g}^{2} v_{h}=v_{g}^{i} v_{h} v_{g} v_{h}, h g^{2} h=g^{i} h g h$ and $\quad h g h^{-1}=g^{i} \quad(1 \leq i \leq n-1)$;

(A2) $v_{h} v_{g}^{2} v_{h}=v_{h} v_{g}^{i} v_{h} v_{g}, h g^{2} h=h g^{i} h g$ and $h g h^{-1}=g^{2-i} \quad(1 \leq i \leq n-1)$;

(A3) $v_{h} v_{g}^{2} v_{h}=v_{g}^{i} v_{h}, h g^{2} h=g^{i} h^{2}$ and $\quad h g^{2} h^{-1}=g^{i} \quad(1 \leq i \leq n-1)$.

This shows that $\left\langle g^{2}\right\rangle$ is a normal cyclic subgroup of $G$.

If $g$ is a 2-element of $G$, then $1+v_{g}$ is nilpotent and by Lemma 2 of [6] we deduce that $1+v_{g}$ is a central element of $K_{\rho} G$. Therefore $v_{g} v_{h}=v_{h} v_{g}$ for every $h \in G$.

If $g$ is an element of odd order, then $\left\langle g^{2}\right\rangle=\langle g\rangle$ and from (A1), (A2) and (A3) we obtain that every cyclic subgroup of $G$ is normal, i.e. $G$ is either abelian, or hamiltonian. Since the 2-elements of $G$ are central, we conclude that $G$ is an abelian torsion group, i.e. condition (i) holds. Now by (A1) and (A2) it follows that $i=1$ and $v_{g} v_{h}=v_{h} v_{g}$. In case (A3) we have $i=2$ and $v_{h} v_{g}^{2}=v_{g}^{2} v_{h}$. But $\left\langle v_{g}^{2}\right\rangle=\left\langle v_{h}\right\rangle$, so $v_{h}$ commutes with $v_{g}^{i}$ for all $i=1, \ldots, n-1$. Therefore condition (ii) also holds.

Now, suppose that $\operatorname{char}(K) \neq 2$. Then by (3), we conclude that for the product $v_{h} v_{g} v_{h}$ we have the following four cases:

(B1) $v_{h} v_{g} v_{h}=v_{g}^{i} v_{h}^{2}, h g h=g^{i} h^{2}$ and $h g h^{-1}=g^{i} \quad(1 \leq i \leq n-1)$;

(в2) $v_{h} v_{g} v_{h}=v_{h} v_{g}^{i} v_{h} v_{g}, h g h=h g^{i} h g$ and $h g h^{-1}=g^{1-i} \quad(0 \leq i \leq n-1)$;

(в3) $v_{h} v_{g} v_{h}=-v_{h} v_{g}^{i} v_{h}, h g h=h g^{i} h$ and $g^{i-1}=1$, which is impossible, because $2 \leq i \leq n-1$ and $g$ is of order $n$;

(B4) $v_{h} v_{g} v_{h}=-v_{g}^{i} v_{h} v_{g} v_{h}, h g h=g^{i} h g h$ and $g^{i}=1$, which is impossible, because $1 \leq i \leq n-1$.

Therefore $\langle g\rangle$ is a normal cyclic subgroup of $G$ for every $g \in G$. Hence $G$ is either abelian or a hamiltonian group.

Assume that $G$ is hamiltonian and $\left\langle g, h \mid g^{4}=1, h^{2}=g^{2}, h g h^{-1}=g^{-1}\right\rangle \cong Q_{8}$. Then by (B1) and (B2), it follows that either $i=3$ or $i=2$, respectively. Hence we obtain that $v_{h} v_{g}=v_{g}^{3} v_{h}$, where $v_{g}^{4}=v_{h}^{4}=1$.

Let $(\alpha, \beta)$ be a nontrivial solution of the equation $x^{2}+y^{2}=0$ in $K$. Then as in the proof of Theorem 1 we establish that

$$
w=\alpha\left(v_{g}^{2} v_{h}-v_{h}\right)+\beta\left(v_{g}^{3} v_{h}-v_{g} v_{h}\right)
$$

is a nonzero nilpotent element of $K_{\rho} G$ with $z^{2}=0$. Therefore $w$ is a central element of $K_{\rho} G$. But $w v_{h} \neq v_{h} w$, so we obtain a contradiction. Thus $G$ is abelian and condition (i) holds. If $g h=h g$, then by (B1) and (B2) it follows that either $i=1$ or $i=2$, respectively. Hence we obtain that $v_{h} v_{g}=v_{g} v_{h}$ for all $g, h \in G$ and so condition (ii) also follows.

Conversely, if the conditions (i) and (ii) hold, then $K_{\rho} G$ is a commutative ring. For every element $a \in K_{\rho} G$ with $H=\langle\operatorname{Supp}(a)\rangle$, the ring $K_{\rho} H$ is artinian and 
$R \cong K_{\rho} H / \mathfrak{N i l}\left(K_{\rho} H\right)$ is a finite sum of fields. Therefore $R$ is strongly regular and hence $K_{\rho} H$ is a $\xi N$-ring. Since $a \in K_{\rho} H$, we deduce that $K_{\rho} G$ is a $\xi N$-ring.

Note that if $K_{\rho} G$ is a $\xi N$-ring, then the periodicity of $G$ can be proved directly. Indeed, if $g \in G$ is an element of infinite order and $z=\left(u_{g}-1\right)^{2} x-\left(u_{g}-1\right)$ is a central nilpotent element of $K_{\rho} G$, then $z^{n}=0$ for some $n \geq 1$. By Corollary 1 we deduce that $\left[\left(u_{g}-1\right) x-1\right] z^{n-1}=0$.

Using the fact that $z$ is central, we can prove by induction that

$$
\left[\left(u_{g}-1\right) x-1\right]^{k} z^{n-k}=0
$$

for every $k \geq 1$. Therefore $\left[\left(u_{g}-1\right) x-1\right]^{n}=0$. This equality shows that $u_{g}-1$ is right invertible in $K_{\rho} G$, which again is impossible by Corollary 1

\section{REFERENCES}

[1] A. A. Bovdi. Crossed products of a semigroup and a ring. Dokl. Akad. Nauk SSSR, 137:1267$1269,1961$.

[2] A. A. Bovdi and T. P. Lángi. On the regularity of group algebras. Arch. Math. (Brno), 32(2):117-121, 1996.

[3] I. G. Connell. On the group ring. Canad. J. Math., 15:650-685, 1963.

[4] V. Gupta. A generalization of strongly regular rings. Acta Math. Hungar., 43(1-2):57-61, 1984.

[5] M. Kargapolov and I. Merzliakov. Éléments de la théorie des groupes. Traduit du Russe: Mathématiques. [Translations of Russian Works: Mathematics]. "Mir", Moscow, 1985. Translated from the Russian by V. Kotliar.

[6] W. S. Martindale, III. The structure of a special class of rings. Proc. Amer. Math. Soc., 9:714-721, 1958.

[7] S. V. Mihovski. On the stongly regular group rings. Bull. Inst. Math. Bulg. Acad. Sci., 14:6771, 1970.

[8] D. S. Passman. Radicals of twisted group rings. Proc. London Math. Soc. (3), 20:409-437, 1970.

[9] D. S. Passman. Radicals of twisted group rings. II. Proc. London Math. Soc. (3), 22:633-651, 1971.

[10] D. S. Passman. The algebraic structure of group rings. Pure and Applied Mathematics. WileyInterscience [John Wiley \& Sons], New York, 1977.

[11] A. Rakhnev. Some properties of group $\xi$-rings. Plovdiv. Univ. Nauchn. Trud., 21(1):13-24, 1983.

[12] Y. Utumi. On $\xi$-rings. Proc. Japan Acad., 33:63-66, 1957.

Department of Math. Sciences, UAE University - Al-Ain, United Arab Emirates

E-mail address: vbovdi@gmail.com

Department of Algebra, University of Plovdiv, Bulgaria

E-mail address: mihovski@uni-plovdiv.bg 